\title{
Cross Sections and Asymmetry Parameters for
} Formic Acid in the Vacuum-Ultraviolet Energy

\section{Range}

M. M. Fujimoto, ${ }^{\dagger}$ H. K. Tanaka,,$\uparrow$ R. R. T. Marinho, ${ }^{\S}$ A. Medina, $\|$ F. V. Prudente, $₫$ and M. G. P. Homem ${ }^{*, \perp}$

$\dagger$ †epartamento de Física, Universidade Federal do Paraná, 81.531-980, Curitiba, PR, Brazil.

$\ddagger$ Instituto Federal da Bahia, 45.810-000, Porto Seguro, BA, Brazil.

【Instituto de Física, Universidade Federal da Bahia, 40.170-115, Salvador, BA, Brazil.

§Instituto de Física, Universidade de Brasília, Box 4455, 70910-970, Brasilia, DF, Brazil.

|| Instituto de Física, Universidade Federal do Rio de Janeiro, 21941-972, Rio de Janeiro,

$$
\text { RJ, Brazil. }
$$

$\perp$ Departamento de Química, Universidade Federal de São Carlos, 13.565-905, São Carlos, SP, Brazil.

E-mail: mghomem@ufscar.br 
Table S1. Experimental ionization quantum yields $(\eta)$, photoabsorption $\left(\sigma_{a}\right)$, photoionization $\left(\sigma_{i}\right)$ and neutral-decay $\left(\sigma_{n}\right)$ cross sections for formic acid. Cross sections are given in units of $\mathrm{Mb}$. An overall uncertainty of $10 \%$ was determined in the $\eta$ values, $4 \%$ in the $\sigma_{a}$, and $11 \%$ in the $\sigma_{i}$ and $\sigma_{n}$.

Table S2. Partial ionization cross sections for the ions $\mathrm{CO}^{+}(m / q=28), \mathrm{COH}^{+}(m / q=29)$, $\mathrm{HCOH}^{+}(m / q=30), \mathrm{CO}_{2}^{+}(m / q=44), \mathrm{COOH}^{+}(m / q=45), \mathrm{HCOOH}^{+}(m / q=46)$, and $(\mathrm{HCOOH}) \mathrm{H}^{+}(m / q=47)$ at some selected energies. The data are given in units of $\mathrm{Mb}$ and were obtained from the present $\sigma_{i}$ and the previously reported partial ion-yield of formic acid of Arruda et al. ${ }^{11}$ The overall experimental uncertainty was determined in $15 \%$.

Figure S1. $(d f / d E) / E^{2}$ in function of photon energy for formic acid. Below 0.4 a.u. $(10.8 \mathrm{eV})$ the data were obtained using the $\sigma_{a}$ of Leach et al. ${ }^{9}$; above 0.8 a.u. $(21.5 \mathrm{eV})$ the data were extrapolated using the asymptotic behavior $\sigma_{a} \propto E^{-3.5}$. The numerical integration over the entire energy range gives a value of $(22.0 \pm 0.7)$ a.u. for the static dipole polarizability.

Figure S2. Present theoretical photoionization cross sections for formic acid obtained in the dipole-velocity $(V)$ form. (.....$),\left(10 a^{\prime}\right)^{-1}$ state; $(----),\left(2 a^{\prime \prime}\right)^{-1}$ state; $(---),\left(9 a^{\prime}\right)^{-1}$ state; $(-.-),.\left(1 a^{\prime \prime}\right)^{-1}$ state; $(-.-),.\left(8 a^{\prime}\right)^{-1}$ state; $(-. .),.\left(7 a^{\prime}\right)^{-1}$ state; (--.-), summed cross sections; (-), present experimental data; (-) experimental results deducted from refs. 4 and 9 . 
Table S1. Experimental ionization quantum yields $(\eta)$, photoabsorption $\left(\sigma_{a}\right)$, photoionization $\left(\sigma_{i}\right)$ and neutral-decay $\left(\sigma_{n}\right)$ cross sections for formic acid. Cross sections are given in units of $\mathrm{Mb}$. An overall uncertainty of $10 \%$ was determined in the $\eta$ values, $4 \%$ in the $\sigma_{a}$, and $11 \%$ in the $\sigma_{i}$ and $\sigma_{n}$.

\begin{tabular}{cccccccccc}
\hline \hline $\mathrm{E}(\mathrm{eV})$ & $\eta$ & $\sigma_{a}$ & $\sigma_{i}$ & $\sigma_{n}$ & $\mathrm{E}(\mathrm{eV})$ & $\eta$ & $\sigma_{a}$ & $\sigma_{i}$ & $\sigma_{n}$ \\
\hline 13.5 & 0.67 & 34.45 & 23.04 & 11.41 & 17.4 & 0.88 & 51.43 & 45.39 & 6.04 \\
13.6 & 0.66 & 35.81 & 23.73 & 12.08 & 17.6 & 0.90 & 52.23 & 46.92 & 5.31 \\
13.8 & 0.64 & 40.62 & 25.94 & 14.68 & 17.8 & 0.93 & 52.74 & 49.20 & 3.54 \\
14.0 & 0.63 & 44.16 & 27.95 & 16.22 & 18.0 & 0.96 & 52.35 & 50.30 & 2.05 \\
14.2 & 0.65 & 45.53 & 29.38 & 16.15 & 18.2 & 0.97 & 52.02 & 50.65 & 1.37 \\
14.4 & 0.67 & 46.46 & 31.34 & 15.12 & 18.4 & 0.99 & 51.42 & 50.74 & 0.69 \\
14.6 & 0.72 & 45.86 & 32.79 & 13.07 & 18.6 & 0.99 & 50.79 & 50.37 & 0.42 \\
14.8 & 0.77 & 41.15 & 31.74 & 9.41 & 18.8 & 1.00 & 50.61 & 50.65 & 0.00 \\
15.0 & 0.82 & 38.26 & 31.26 & 7.00 & 19.0 & 1.00 & 50.57 & 50.57 & 0.00 \\
15.2 & 0.85 & 37.05 & 31.66 & 5.40 & 19.2 & 1.00 & 50.71 & 50.71 & 0.00 \\
15.4 & 0.88 & 37.44 & 33.10 & 4.34 & 19.4 & 1.00 & 51.19 & 51.19 & 0.00 \\
15.6 & 0.91 & 38.22 & 34.77 & 3.45 & 19.6 & 1.00 & 51.67 & 51.67 & 0.00 \\
15.8 & 0.93 & 38.90 & 36.24 & 2.65 & 19.8 & 1.00 & 51.70 & 51.70 & 0.00 \\
16.0 & 0.92 & 41.58 & 38.05 & 3.53 & 20.0 & 1.00 & 51.47 & 51.47 & 0.00 \\
16.2 & 0.91 & 43.55 & 39.49 & 4.07 & 20.2 & 1.00 & 51.68 & 51.68 & 0.00 \\
16.4 & 0.89 & 43.65 & 38.82 & 4.83 & 20.4 & 1.00 & 51.75 & 51.75 & 0.00 \\
16.6 & 0.87 & 45.27 & 39.61 & 5.66 & 20.6 & 1.00 & 51.49 & 51.49 & 0.00 \\
16.8 & 0.86 & 47.88 & 40.99 & 6.89 & 20.8 & 1.00 & 51.75 & 51.75 & 0.00 \\
16.8 & 0.86 & 47.74 & 40.85 & 6.88 & 21.0 & 1.00 & 51.81 & 51.81 & 0.00 \\
17.0 & 0.85 & 48.93 & 41.62 & 7.31 & 21.2 & 1.00 & 51.85 & 51.85 & 0.00 \\
17.2 & 0.86 & 50.33 & 43.40 & 6.92 & 21.4 & 1.00 & 52.00 & 52.00 & 0.00 \\
\hline
\end{tabular}


Table S2. Partial ionization cross sections for the ions $\mathrm{CO}^{+}(m / q=28), \mathrm{COH}^{+}(m / q=29)$, $\mathrm{HCOH}^{+}(m / q=30), \mathrm{CO}_{2}^{+}(m / q=44), \mathrm{COOH}^{+}(m / q=45), \mathrm{HCOOH}^{+}(m / q=46)$, and $(\mathrm{HCOOH}) \mathrm{H}^{+}(m / q=47)$ at some selected energies. The data are given in units of $\mathrm{Mb}$ and were obtained from the present $\sigma_{i}$ and the previously reported partial ion-yield of formic acid of Arruda et al. ${ }^{11}$ The overall experimental uncertainty was determined in $15 \%$.

\begin{tabular}{|c|c|c|c|c|c|c|c|}
\hline \multirow[t]{2}{*}{$\mathrm{E}(\mathrm{eV})$} & \multicolumn{7}{|c|}{$m / q$} \\
\hline & 28 & 29 & 30 & 44 & 45 & 46 & 47 \\
\hline 13.6 & 0.00 & 3.01 & 0.04 & 0.00 & 7.25 & 11.23 & 0.25 \\
\hline 13.8 & 0.00 & 4.21 & 0.06 & 0.00 & 7.48 & 12.01 & 0.21 \\
\hline 14.0 & 0.00 & 5.13 & 0.05 & 0.00 & 7.53 & 12.40 & 0.29 \\
\hline 14.2 & 0.00 & 7.19 & 0.08 & 0.00 & 7.93 & 11.90 & 0.32 \\
\hline 14.4 & 0.00 & 8.68 & 0.10 & 0.00 & 8.37 & 11.60 & 0.28 \\
\hline 14.6 & 0.00 & 9.98 & 0.10 & 0.00 & 8.59 & 11.29 & 0.31 \\
\hline 14.8 & 0.00 & 10.60 & 0.15 & 0.00 & 8.21 & 10.21 & 0.26 \\
\hline 15.0 & 0.00 & 11.01 & 0.09 & 0.00 & 8.08 & 9.58 & 0.25 \\
\hline 15.2 & 0.00 & 11.75 & 0.12 & 0.00 & 7.84 & 9.68 & 0.24 \\
\hline 15.4 & 0.00 & 12.70 & 0.14 & 0.00 & 7.96 & 9.85 & 0.24 \\
\hline 15.6 & 0.01 & 14.30 & 0.18 & 0.01 & 7.94 & 10.09 & 0.24 \\
\hline 15.8 & 0.02 & 15.97 & 0.14 & 0.02 & 8.04 & 9.48 & 0.27 \\
\hline 16.0 & 0.03 & 16.67 & 0.18 & 0.02 & 8.06 & 9.14 & 0.28 \\
\hline 16.2 & 0.06 & 17.73 & 0.19 & 0.05 & 8.55 & 9.57 & 0.24 \\
\hline 16.4 & 0.05 & 18.03 & 0.20 & 0.03 & 8.53 & 9.31 & 0.30 \\
\hline 16.6 & 0.01 & 18.93 & 0.20 & 0.04 & 8.45 & 8.95 & 0.27 \\
\hline 16.8 & 0.09 & 19.89 & 0.23 & 0.12 & 8.82 & 8.81 & 0.24 \\
\hline 17.0 & 0.06 & 20.67 & 0.26 & 0.23 & 8.73 & 8.43 & 0.21 \\
\hline 17.2 & 0.13 & 22.01 & 0.24 & 0.31 & 9.05 & 8.20 & 0.23 \\
\hline 17.4 & 0.13 & 23.23 & 0.25 & 0.37 & 9.54 & 8.12 & 0.16 \\
\hline 17.6 & 0.16 & 24.95 & 0.26 & 0.47 & 9.76 & 7.91 & 0.19 \\
\hline 17.8 & 0.18 & 26.52 & 0.35 & 0.60 & 10.23 & 7.85 & 0.24 \\
\hline 18.0 & 0.16 & 27.11 & 0.34 & 0.59 & 10.45 & 7.75 & 0.24 \\
\hline 18.2 & 0.17 & 27.46 & 0.37 & 0.62 & 10.38 & 7.71 & 0.15 \\
\hline 18.4 & 0.17 & 27.43 & 0.35 & 0.64 & 10.36 & 7.52 & 0.21 \\
\hline 18.6 & 0.21 & 27.44 & 0.42 & 0.65 & 10.32 & 7.43 & 0.15 \\
\hline 18.8 & 0.24 & 27.36 & 0.38 & 0.72 & 10.33 & 7.23 & 0.14 \\
\hline 19.0 & 0.24 & 27.26 & 0.36 & 0.70 & 10.35 & 7.24 & 0.17 \\
\hline
\end{tabular}




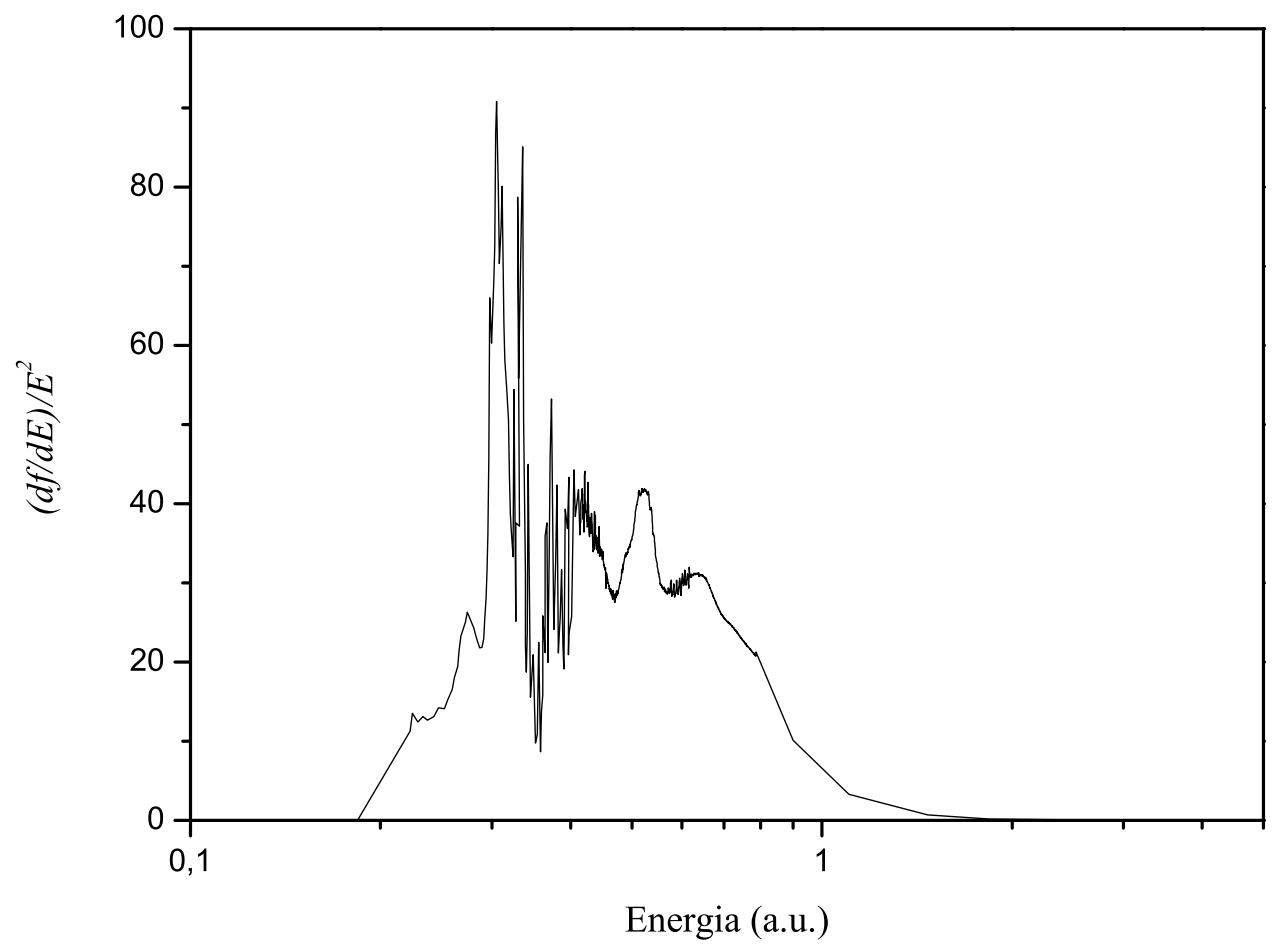

Figure S1. $(d f / d E) / E^{2}$ in function of photon energy for formic acid. Below 0.4 a.u. (10.8 $\mathrm{eV}$ ) the data were obtained using the $\sigma_{a}$ of Leach et al. ${ }^{9}$; above 0.8 a.u. $(21.5 \mathrm{eV})$ the data were extrapolated using the asymptotic behavior $\sigma_{a} \propto E^{-3.5}$. The numerical integration over the entire energy range gives a value of $(22.0 \pm 0.7)$ a.u. for the static dipole polarizability. 


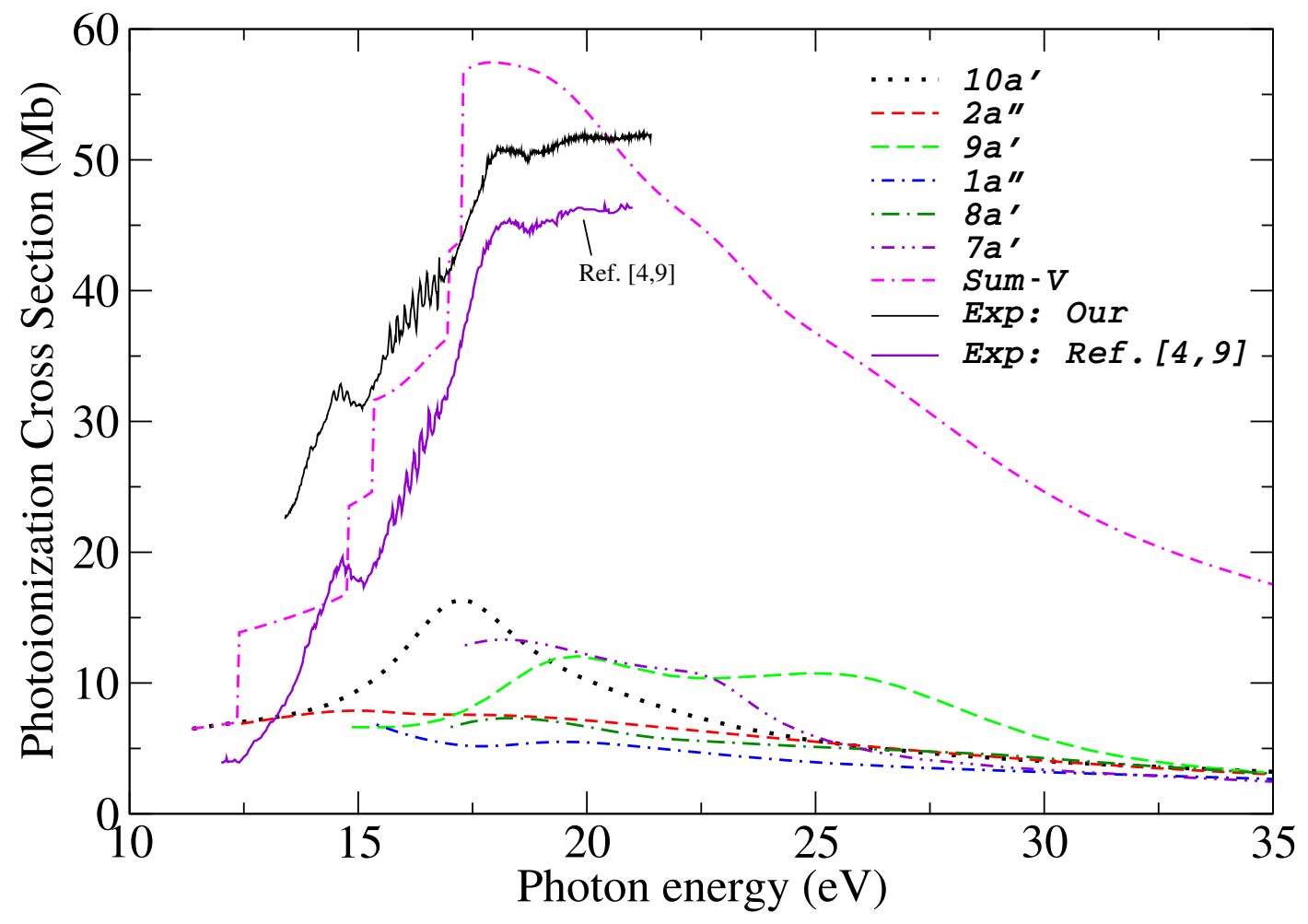

Figure S2. Present theoretical photoionization cross sections for formic acid obtained in the dipole-velocity $(V)$ form. $(\cdots \cdots),\left(10 a^{\prime}\right)^{-1}$ state; $(---),\left(2 a^{\prime \prime}\right)^{-1}$ state; $(---),\left(9 a^{\prime}\right)^{-1}$ state; $(-. .-),\left(1 a^{\prime \prime}\right)^{-1}$ state; (-.-.), $\left(8 a^{\prime}\right)^{-1}$ state; $(-. .),.\left(7 a^{\prime}\right)^{-1}$ state; (--.-), summed cross sections; $(-)$, present experimental data; (-) experimental results deducted from refs. 4 and 9. 